
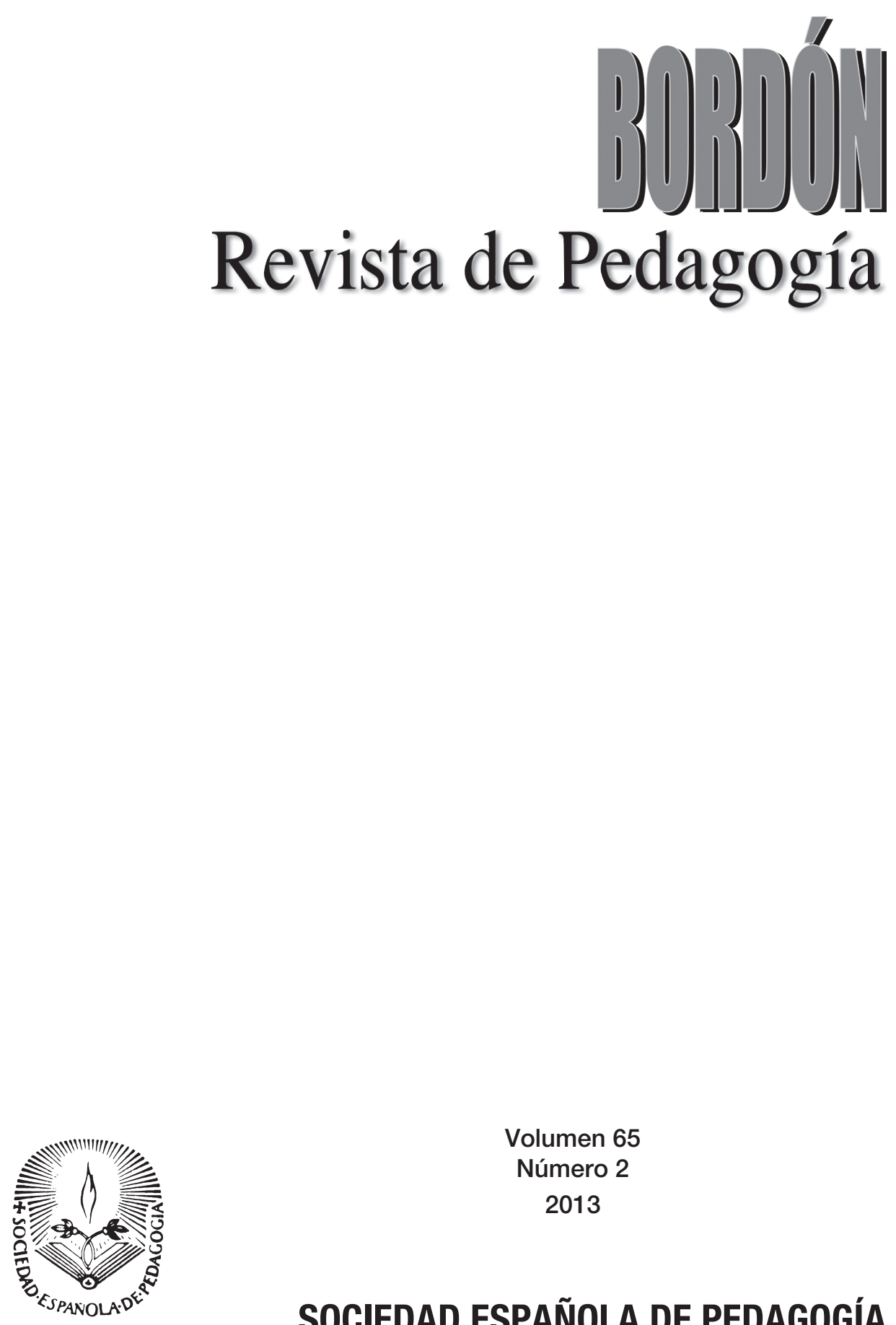

Volumen 65

Número 2

2013

SOCIEDAD ESPAÑOLA DE PEDAGOGÍA 



\title{
AUTOEUALUACIÓn DE COMPETENCIAS InFORMACIONALES EN EDUCACION SECUNDARIA: PROPUESTA DE MODELO CAUSAL DESDE UNA PERSPECTIUA DE GÉnERO
}

\section{Information skills self-assessment in secondary education. f causal model proposal from a gender perspective}

\author{
Ma JOSÉ RODRÍGUEZ CONDE \\ SUSANA OLMOS MIGUELÁÑEZ \\ FERNANDO MARTÍNEZ ABAD \\ Universidad de Salamanca
}

INTRODUCCIÓN. La inclusión en los currículos de educación básica de competencias clave relacionadas con el manejo de la información, responde a la transformación social relacionada con el impacto de las tecnologías de la información y la comunicación. Por otra parte, proliferan estudios de género que evidencian diferencias por sexo en la adquisición de habilidades informáticas. Dada la estrecha relación entre las competencias informacionales y estas tecnologías, el presente estudio tiene como objetivo analizar las diferencias de género en el desarrollo de las citadas competencias a partir del diseño de un modelo causal. MÉTODO. Se utiliza metodología correlacional mediante modelos causales a través de estudios de cuestionario de autoinforme. Se aplican sendas escalas para medir la competencia informacional autopercibida y la frecuencia y nivel en el empleo de herramientas informáticas a una muestra representativa de 1.162 sujetos, estudiantes de entre 14 y 16 años de Castilla y León (España). RESULTADOS. Se obtienen buenos indicadores de ajuste para el modelo causal en ambos grupos. El contraste de las relaciones planteadas en el modelo indica que el efecto que ejerce el perfil de manejo de herramientas informáticas en el hogar (entornos educativos no formales e informales), sobre el desarrollo de competencias informacionales, es diferente en función del género. DISCUSIÓN. Se concluye señalando la importancia de estudiar y definir estos diferentes perfiles en el manejo de herramientas informáticas, dada su influencia en el aprendizaje de las competencias informacionales.

Palabras clave: Tecnologías de la información y la comunicación, Habilidades de información, Educación informal, Modelos de ecuaciones estructurales, Estudios de género. 


\section{Introducción}

En un entorno mediado por las tecnologías de la información y la comunicación (TIC), en el que todas las relaciones y sectores sociales están determinados por dichas tecnologías (Castells, 1999; Valimaa y Hoffman, 2008), la sociedad actual se encuentra con nuevas necesidades formativas que requieren una renovación de los objetivos y competencias, contenidos, metodologías y procesos de evaluación de nuevos aprendizajes (Jones y Goff, 2011).

Por su parte, los entornos de educación no formal e informal, más flexibles y adaptables, parecen suplir estas lagunas existentes en el contexto de una educación formal inmersa en un entorno tan cambiante e incierto como la «sociedad líquida» (Bauman, 2005).

Sin embargo, desde los responsables en materia de diseño curricular nacional o autonómico, ya se han comenzado a completar algunos de los pasos previos para adaptar los currículos a la nueva realidad social. Fruto de ello es la renovación de los procesos de enseñanza-aprendizaje, desde enfoques por objetivos, centrados en el objeto a enseñar, a los actuales enfoques de formación por competencias, más enfocados en el proceso de aprendizaje que se lleva a cabo (Calvo Bernardino y Mingorance Arnáiz, 2010; de Miguel Díaz, 2010).

Por otro lado, en cuanto a los contenidos, tanto a escala nacional como internacional, se han integrado como competencias básicas ejes transversales de la educación obligatoria, competencias relacionadas con el manejo de la información en redes informáticas. Así, surgen las competencias informacionales como un conjunto de competencias esenciales a desarrollar por todos los ciudadanos de esta sociedad de la información (Weiner, 2010).

Desde la perspectiva de esta investigación, inmersa en un proyecto nacional I+D financiado por el actual Ministerio de Economía y Competitividad de España (ref.: EDU200908753), se definen las competencias informacionales conforme a la propuesta de la Conferencia de Rectores de las Universidades Españolas (CRUE), junto con la Red de Bibliotecas Universitarias (REBIUN), que señalan lo siguiente:

«Las competencias informacionales son el conjunto de conocimientos, habilidades, disposiciones y conductas que capacitan a los individuos para reconocer cuándo necesitan información, dónde localizarla, cómo evaluar su idoneidad y darle el uso adecuado de acuerdo con el problema que se plantea» (2009: 5).

Conforme a esta definición, y a numerosos estudios teóricos y empíricos llevados a cabo a este respecto (Catts y Lau, 2009; Mackey y Jacobson, 2011; Rodríguez Conde, Olmos Migueláñez, Pinto Molina, Martínez Abad, y García Riaza, 2011), las competencias informacionales se consideran generalmente como un constructo formado por cuatro dimensiones teóricas o subcompetencias. Estas dimensiones se definen como la capacidad de la persona para buscar la información que necesita (dimensión de búsqueda de la información); para analizar y seleccionar la información localizada de manera eficiente (dimensión de selección de la información); para organizar y procesar la información de manera apropiada (dimensión de procesamiento de la información); y para utilizar y comunicar la información de modo eficaz, ética y legalmente y con el fin e colaborar en una construcción común del conocimiento (dimensión de comunicación y difusión de la información). En todos los casos, estas dimensiones están en función de las necesidades informacionales concretas de la situación en la que está inmerso el sujeto.

Una vez señalada la evidencia de la importancia de las TIC, como factor determinante en el surgimiento y desarrollo de la sociedad de la información, estamos en condiciones de remarcar también que en el ámbito educativo su impacto 
ha sido el principal causante de la aparición y definición de las competencias informacionales, como pilar fundamental para alcanzar el objetivo de la sociedad del conocimiento. Así, la amplia literatura existente al respecto aporta evidencias acerca de la estrecha relación existente entre las TIC y el desarrollo de estas competencias (Jarson, 2010; O'Farrill, 2008; Pifarré, Sanuy, Vendrell y Gòdia, 2009).

Por otro lado, se detecta en la investigación educativa un interés creciente hacia los estudios de género (Gargallo-Castel, Esteban-Salvador y Pérez Sanz, 2010; Mullola et al., 2011; Ohland et al., 2011), que tratan de localizar algunas diferencias culturales existentes entre hombres y mujeres que tengan algún tipo de efecto sobre las condiciones en las que se desarrollan ciertas competencias. Este tipo de investigaciones es especialmente recurrente en trabajos relacionados con los hábitos de manejo de las TIC, y la evidencia científica muestra que existen diferencias de género significativas en lo que respecta al perfil general en el manejo de estas tecnologías (Iglesias Fernández, Llorente Heras, y Dueñas Fernández, 2010; Ruiz Palomero y Sánchez Rodríguez, 2010).

Centrándonos más concretamente en la investigación que aquí nos ocupa, la revisión del estado de la cuestión lleva a preguntarnos sobre si estas diferencias de género en cuanto al perfil de manejo de las TIC tendrán algún tipo de relación sobre la percepción que los estudiantes de educación secundaria tienen sobre el aprendizaje adquirido de las competencias informacionales y, por ende, si la manera en la que el manejo de las TIC colabora en el desarrollo de las competencias informacionales es diferente en hombres y en mujeres.

\section{Metodología de la investigación}

Partiendo de los supuestos teóricos planteados, se plantea como objetivo de la investigación el siguiente: identificar diferencias debidas al género entre estudiantes de Educación Secundaria Obligatoria, en cuanto a la autopercepción sobre el desarrollo de competencias informacionales, mediadas por los diversos hábitos y niveles en lo que respecta al manejo de herramientas informáticas. El propósito último es aportar información empírica que pueda favorecer algún tipo de toma de decisiones en los centros educativos. Por tanto, se intenta proporcionar información válida y fiable para colaborar en la explicación razonable de los fenómenos objeto de estudio (competencias informacionales), a fin de contribuir a la configuración de un cuerpo de conocimientos sólido en este nuevo ámbito de estudio para el pedagogo.

\section{Diseño de la investigación}

El presente estudio propone un diseño de investigación de tipo inferencial y correlacional basado en modelos causales (Mateo Andrés, 2004), a través de estudios de cuestionario de autoinforme (Kerlinger y Lee, 2002 y Fernández Ballesteros, 2004). A través del diseño planteado, se pretende contrastar la siguiente hipótesis: $L a$ frecuencia y el nivel en el manejo de herramientas $y$ aplicaciones informáticas ejercen un efecto diferente sobre el nivel autopercibido en competencias informacionales, en función del sexo.

Se puede englobar la investigación dentro de las metodologías ex-post-facto, ya que no modificamos directamente las variables objeto de estudio, sino que simplemente buscamos localizar las relaciones existentes entre las mismas.

\section{Variables e instrumentos}

Dentro del estudio planteado, y en función de la hipótesis general planteada, se identifica como «variable predoctora» el sexo de los sujetos que conforman la muestra, y como «variable criterio» el nivel autopercibido sobre competencias informacionales. Se pone en contraste cómo las diferencias en la frecuencia y nivel en el manejo 
de herramientas informáticas debidas al género, entre los estudiantes de educación secundaria, influyen sobre la adquisición de competencias informacionales. Así, a partir del modelo de ecuaciones estructurales planteado, se pueden identificar tres «variables exógenas»: nivel en el manejo de herramientas informáticas, frecuencia en el empleo de herramientas informáticas en la escuela (entorno de educación formal) y frecuencia en el empleo de herramientas informáticas en el hogar (entorno de educación no formal). Por otra parte, la «variable endógena» del estudio se corresponde con el nivel autopercibido sobre competencias informacionales.

Para medir el nivel autopercibido sobre competencias informacionales se aplica un cuestionario adaptado de Pinto (2009). A partir de dicho cuestionario, se obtienen 19 ítems, que conforman el constructo que se pretende medir, y que se definen a partir de una escala «tipo Likert» (Morales Vallejo, Urosa, y Blanco, 2003) con puntuaciones que se sitúan entre 1 (poco competente) y 9 (muy competente). Los sujetos deben situarse para cada ítem en un valor en la escala que les identifique con su nivel de competencia autopercibido. La estructura dimensional de dicho instrumento, que se define en cuatro factores, ha sido validada tanto a nivel teórico (Pinto, 2009), como factorial (Rodríguez Conde, Olmos Migueláñez, y Martínez Abad, 2012) mediante metodología de ecuaciones estructurales (Jornet, González-Such y Perales, 2012).

Para valorar la frecuencia y el nivel en el empleo, se aplican sendas escalas de frecuencia y de cantidad, con puntuaciones de rangos 1 a 3 ( 1 , menos de una vez al mes; 2 , entre una y cuatro veces al mes; 3 , varias veces por semana) y 1 (nulo) a 5 (alto), respectivamente.

\section{Población y selección de la muestra}

Para la presente investigación se define una población compuesta por todos los estudiantes de la Comunidad Autónoma de Castilla y León
(España) que, en el momento de la aplicación de la encuesta (mayo y junio de 2009), cursan tercero y cuarto de Educación Secundaria Obligatoria (ESO) en centros financiados con fondos públicos (estudiantes entre 14 y 16 años, en su mayoría).

A partir de la población definida para el estudio, se procedió a la aplicación de un método de muestreo aleatorio por conglomerados para la selección de una muestra representativa. Partiendo de la consideración de máxima variabilidad ( $p=q=0.5)$, con un nivel de confianza del 95\% y un error definido de $\pm 3 \%$, la muestra mínima necesaria resulta de 1.087 sujetos repartidos en 13 centros educativos seleccionados al azar de entre los 381 conglomerados identificados.

La muestra final obtenida es de 1.162 sujetos, de los cuales, debido a la existencia de datos perdidos que reducen el número de casos válidos, nos encontramos, para llevar a cabo este estudio en concreto, con un total de $1.011 \mathrm{su-}$ jetos. En concreto, contamos en la muestra con 504 hombres $(49,85 \%)$ y 507 mujeres $(50,15 \%)$. Esta paridad de casos en lo que respecta al sexo informa sobre una tendencia que indica que los resultados se ajustan a la distribución de frecuencias de la población y no existen sesgos muestrales en lo que respecta a la variable sexo.

\section{Análisis de datos}

En primer lugar, se procede al contraste de los supuestos previos al análisis. Para ello, a modo exploratorio, se lleva a cabo el análisis de los valores de asimetría y curtosis, así como el cálculo del Coeficiente de Mardia, que prueba la normalidad multivariante (Bollen, 1989). Por otro lado se verifican las condiciones de orden y de rango.

A continuación se aplica la técnica inferencial no paramétrica de la U de Mann-Whitney en función del sexo, sobre cada una de las variables intervinientes en el modelo causal. Paralelamente, 
se calcula el tamaño del efecto a partir del estadístico r (Corder y Foreman, 2009), calculado del valor del estadístico de contraste obtenido en la prueba de la U de Mann-Whitney. Se pretende así estudiar la influencia directa que puedan ejercer estas variables sobre las diferencias intergrupo localizadas en las relaciones planteadas en el modelo de ecuaciones estructurales.

En la implementación del modelo causal, una vez contrastados los supuestos previos de normalidad (Kline, 2005) y comprobado el cumplimiento de las condiciones de orden y rango (identificación del modelo). Como el tamaño de la muestra es mayor a 1.000 sujetos (Muthén y Kaplan, 1985), se decide aplicar el método de estimación no paramétrico asintóticamente libre de distribución (Browne, 1984; Hawkins, 1982). La bondad de ajuste del modelo se comprueba analizando los indicadores, tanto de ajuste global como incremental.

En la aplicación de todas las técnicas estadísticas se empleó el paquete estadístico IBM SPSS statistics v.19, junto con su complemento AMOS v.19 (licencia campus de la Universidad de Salamanca).

\section{Resultados}

\section{Estudio de los supuestos previos}

Como paso previo a la aplicación de las técnicas propias de los modelos de ecuaciones estructurales, se pone a prueba el cumplimiento de los supuestos previos que tienen que ver con la normalidad de las variables. Los resultados obtenidos en las pruebas de asimetría y curtosis (tabla 1) muestran cómo las variables no se comportan individualmente, a nivel general, conforme a una distribución normal. Por otro lado, a nivel multivariante tampoco existe normalidad, como nos indica el coeficiente de Mardia. Así, se deberá proceder a la aplicación de las técnicas no paramétricas para la prueba del modelo causal planteado.

TABLA 1. Test de normalidad para las variables incluidas en el modelo

Variables Asimetría T Curtosis $\mathrm{T}$

\begin{tabular}{|c|c|c|c|c|}
\hline Frecuencia en el manejo de herramientas informáticas en la escuela & 0.19 & 2.52 & -0.85 & $-5.57 *$ \\
\hline Hombres & 0.17 & 1.58 & -0.90 & $-4.12 *$ \\
\hline Mujeres & 0.18 & 1.61 & -0.90 & $-4.15^{*}$ \\
\hline Frecuencia en el manejo de herramientas informáticas en casa & 0.26 & $3.37 *$ & -0.37 & $-2.44^{*}$ \\
\hline Hombres & 0.26 & $2.36^{*}$ & -0.50 & $-2.31^{*}$ \\
\hline Mujeres & 0.22 & $1.98^{*}$ & -0.31 & -1.42 \\
\hline Habilidades en el manejo de herramientas informáticas & -0.63 & $-8.26^{*}$ & 3.14 & $20.53 *$ \\
\hline Hombres & -0.40 & $-3.67^{*}$ & 3.94 & $18.10^{*}$ \\
\hline Mujeres & -1.02 & $-9.43^{*}$ & 1.21 & $5.55^{*}$ \\
\hline Búsqueda de la información & -0.45 & $-5.83 *$ & 0.18 & 1.20 \\
\hline Hombres & -0.50 & $-4.57^{*}$ & 0.18 & 0.83 \\
\hline Mujeres & -0.31 & $-2.86^{*}$ & -0.09 & -0.42 \\
\hline Selección de la información & -0.25 & $-3.33^{*}$ & 0.33 & $2.14 *$ \\
\hline Hombres & -0.21 & $-1.96^{*}$ & 0.03 & 0.15 \\
\hline Mujeres & -0.31 & $-2.86^{*}$ & 0.69 & $3.16^{*}$ \\
\hline
\end{tabular}


Tabla 1. Test de normalidad para las variables incluidas en el modelo (cont.)

\begin{tabular}{|c|c|c|c|c|}
\hline Variables & Asimetría & $\mathrm{T}$ & Curtosis & $\mathrm{T}$ \\
\hline Procesamiento de la información & -0.33 & $-4.30^{*}$ & 0.14 & 0.94 \\
\hline Hombres & -0.21 & -1.93 & -0.05 & -0.22 \\
\hline Mujeres & -0.39 & $-3.62 *$ & 0.17 & 0.76 \\
\hline Comunicación y difusión de la información & -0.64 & $-8.38^{*}$ & 0.59 & $3.85^{*}$ \\
\hline Hombres & -0.53 & $-4.84^{*}$ & 0.44 & $2.00^{*}$ \\
\hline Mujeres & -0.69 & $-6.36^{*}$ & 0.67 & $3.10^{*}$ \\
\hline Coeficiente de media & & & 11.47 & $16.37^{*}$ \\
\hline
\end{tabular}

Nota: ${ }^{*} \boldsymbol{\alpha}=.05$

Por otro lado, si se analizan las distribuciones de las variables en los grupos de chicos y chicas por separado, se observan tendencias similares en la forma de las funciones de densidad de ambos grupos. En el caso concreto de la variable selección de la información, se aprecian diferencias entre hombres y mujeres en cuanto al apuntamiento de la curva: el nivel autopercibido en cuanto a las competencias de selección de información se concentra en un menor rango de puntuaciones en el caso de las mujeres.

En cuanto a la identificación del modelo, se verifica la condición de orden (11 grados de libertad), la condición de rango (determinante de la matriz de covarianzas $=0.546$ ) y la inexistencia de varianzas/covarianzas del error negativas, errores estándar excesivamente elevados o correlaciones entre los coeficientes estimados muy elevadas.

\section{Estudio inferencial}

Explorando las diferencias obtenidas entre los hombres y las mujeres se pueden observar diferencias, muy ligeras, favorables en todos los casos a los hombres. Para estudiar si estas diferencias son significativas, veamos la tabla 2 , en la que se indican los coeficientes obtenidos en el contraste de hipótesis de diferencia de medias para grupos independientes.

Tabla 2. U de Mann-Whitney, en función del sexo

V. explicativas

$$
\sum \bar{x}_{n} \sum \bar{x}_{m}
$$

$\mathrm{U}$

Z p.

T. E.

\begin{tabular}{lcccccc}
$\begin{array}{l}\text { Frecuencia en el manejo de herramientas } \\
\text { informáticas en la escuela }\end{array}$ & 524.97 & 487.14 & 118204.0 & -2.07 & .04 & 0.07 \\
\hline $\begin{array}{l}\text { Frecuencia en el manejo de herramientas } \\
\text { informáticas en casa }\end{array}$ & 416.17 & 487.14 & 122638.0 & -1.11 & .27 & 0.03 \\
\hline $\begin{array}{l}\text { Habilidades en el manejo de herramientas } \\
\text { nabrar }\end{array}$ & 512.99 & 499.06 & 124243.5 & -0.76 & .45 & 0.02
\end{tabular}

informáticas 
En el contraste de hipótesis, se puede observar cómo, para un valor $\boldsymbol{\alpha}=0.05$, rechazamos la hipótesis nula, en lo que respecta a la frecuencia en uso de herramientas informáticas en el propio centro educativo, en entornos educativos formales. Sin embargo, si analizamos el valor del tamaño del efecto (T. E.), vemos cómo en este caso, al igual que en el resto, se considera bajo. Así, podemos afirmar que las diferencias entre hombres y mujeres en lo que respecta al uso de herramientas informáticas en el centro educativo, aunque son significativas, son pequeñas. En el resto de variables ni siquiera existen diferencias estadísticamente significativas ( $\boldsymbol{\alpha}=0.05)$. El comportamiento de estos adolescentes, considerando el género, parece similar en relación con la variable: uso de los recursos informáticos.

\section{Modelo causal}

En cuanto al modelo causal planteado para hombres y mujeres, la tabla 3 muestra cómo los coeficientes de ajuste global, incremental y de parsimonia de los índices analizados parecen indicar un buen ajuste general en todos los aspectos.

El ajuste global del modelo muestra valores del estadístico de contraste Chi cuadrado que indican una falta de ajuste global $(\mathrm{p}<0.01)$. Sin embargo, estos resultados son esperables, dada la sensibilidad de este tipo de contrastes cuando los tamaños de muestra son elevados (Backhoff Escudero y González-Montesinos, 2012). Por su parte, el índice de bondad de ajuste global GFI, que no depende en el cálculo del tamaño de la muestra (Kline, 2005), muestra valores que indican un buen ajuste (GFI>0.95).

Por otro lado, los índices de ajuste incremental alcanzan en todos los casos valores aceptables (Kline, 2005). En primer lugar, se obtienen valores, tanto en hombres como en mujeres, superiores al mínimo exigible de 0.90 en el índice Comparative Fit Index (CFI), índice habitualmente empleado, ya que compara varios coeficientes de ajuste del modelo. Por otro lado, se obtienen valores también aceptables en el Incremental Fit Index (IFI), más consistente que el CFI, ya que tiene en cuenta en su cálculo los grados de libertad del modelo (Schmidt, Barreyro y Maglio, 2012), y en el Adjusted Goodness of Fit Index (AGFI). Por último, los valores de la correlación múltiple al cuadrado o coeficiente de determinación $\left(\mathrm{R}^{2}\right)$ de la variable latente Competencia informacional autopercibida rondan el 0.30 en todos los casos, siendo ligeramente superior en hombres que en mujeres.

Centrándose en las relaciones individuales planteadas en ambos modelos (figura 1), los datos muestran valores similares en la mayor parte de las vías. Se puede observar, no obstante, una ligera tendencia favorable a los hombres a nivel general, excepto en efecto sobre la competencia informacional, que aportan las habilidades en el manejo de herramientas informacionales, siendo favorable a las mujeres.

TABLA 3. Indicadores de ajuste del modelo de ecuaciones estructurales

\begin{tabular}{|c|c|c|c|c|c|c|c|c|c|}
\hline & \multicolumn{5}{|c|}{ Ajuste global } & \multicolumn{4}{|c|}{ Ajuste incremental } \\
\hline & $\mathrm{X}^{2}$ & g.l. & p. & GFI & RMSEA & CFI & IFI & AGFI & $\mathrm{R}^{2}$ \\
\hline Hombres & 32.715 & 11 & .001 & .979 & .058 & .902 & .906 & .946 & .34 \\
\hline Mujeres & 33.019 & 11 & .001 & .978 & .059 & .899 & .903 & .945 & .28 \\
\hline Multigrupo & 65.734 & 22 & .000 & .979 & .042 & .900 & .905 & 946 & .33 \\
\hline
\end{tabular}


Figura 1. Solución estandarizada de los modelos de ecuaciones estructurales

MODELO HOMBRES

$\begin{array}{llll}.58 & .59 & .69 & .53\end{array}$

Búsqueda Selección Procesamiento Comunicación de de de de información información información información

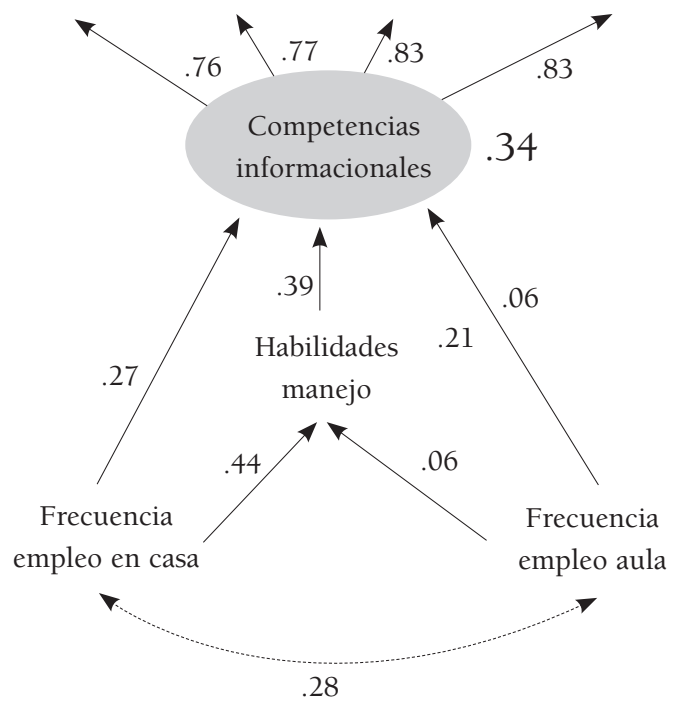

MODELO MUJERES

$\begin{array}{lll}.67 & .58 & .67\end{array}$

.59

Búsqueda Selección Procesamiento Comunicación de de de de información información información información

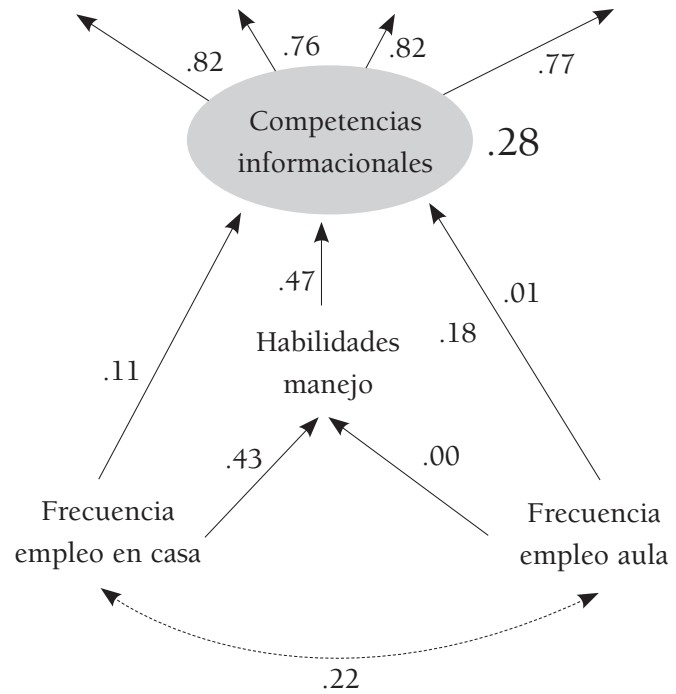

TABla 4. Contraste de hipótesis para la comparación de las vías planteadas por sexo

\begin{tabular}{|c|c|}
\hline $\begin{array}{l}\text { Habilidades } \\
\text { informáticas }\end{array}$ & $\begin{array}{l}\text { Uso en centro } \\
\text { educativo }\end{array}$ \\
\hline
\end{tabular}

Habilidades informáticas

\begin{tabular}{lcccl}
\hline Uso en centro educativo & 1.021 & - & \\
\hline Uso en el hogar & 0.724 & - & - & \\
\hline Competencias informacionales & 1.115 & 1.099 & $2.070^{*}$ & -
\end{tabular}

* Valor de z significativo a un nivel $\boldsymbol{\alpha}=0.05$.

Si nos centramos en las diferencias que más llaman la atención, se puede tener en cuenta que mientras que el modelo en los hombres explica el $34 \%$ de la varianza de la variable endógena competencias informacionales autopercibidas, el modelo en las mujeres explica el 28\%. Por otro lado, se percibe una diferencia importante en el efecto de la frecuencia de empleo de herramientas informáticas en casa (contexto de educación informal) sobre el nivel autopercibido en competencias informacionales. 
Es reseñable el peso de la frecuencia de empleo de herramientas informáticas en el aula sobre las habilidades informáticas y las propias competencias informacionales que es, en todos los casos, muy baja. De hecho, el contraste de hipótesis muestra relaciones no significativas en estas vías.

Por último, en la tabla 4 se analizan las diferencias obtenidas para cada una de las vías planteadas en el modelo.

Los resultados del contraste, mostrados en valores absolutos, indican cómo se obtienen diferencias significativas en la vía correspondiente a los efectos de la frecuencia de empleo de herramientas informáticas en casa sobre las competencias informacionales. Estas diferencias son, como se mostraba en la figura 1, favorables a los hombres.

\section{Discusión y conclusiones}

Partiendo de la premisa de que la formación en la Sociedad de la Información ha adoptado las competencias informacionales como uno de sus pilares esenciales (Mackey y Jacobson, 2011; Weiner, 2010; Williamson, 2009), parece razonable considerar que su estudio y desarrollo se debería promover desde las instituciones educativas. De hecho, en materia de política educativa, se han sucedido pasos importantes dirigidos a impulsar el desarrollo de estas competencias.

Tanto en el marco nacional como internacional, se han integrado las competencias informacionales como parte de las competencias básicas o clave que deben haber sido adquiridas por todas las personas que finalizan la educación básica. Esto queda reflejado en el ámbito europeo en las Recomendaciones del Parlamento Europeo y del Consejo de 18 de diciembre de 2006 sobre las competencias clave para el aprendizaje permanente, que señala la competencia digital como una de las 8 competencias clave y la vincula estrechamente con las TIC. El citado texto define las competencias digitales como «el uso de ordenadores para obtener, evaluar, almacenar, producir, presentar e intercambiar información, y comunicarse y participar en redes de colaboración a través de Internet» (Diario Oficial de la Unión Europea, 2006: 15). En un contexto más cercano, en España, si nos situamos en la etapa educativa de la educación secundaria, en la que se encuentra la población que abarca esta investigación, podemos encontrar la Orden ECI/2220/2007, de 12 de julio, por la que se establece el Currículo y se regula la ordenación de la Educación Secundaria Obligatoria. En dicho documento se plantea como una de las competencias básicas para la etapa de educación secundaria el tratamiento de la información y la competencia digital, que se define como «seleccionar, tratar y utilizar la información y sus fuentes, así como las distintas herramientas tecnológicas» (Boletín Oficial del Estado, 2007: 31690). Se puede observar cómo se vuelven a vincular muy íntimamente las competencias informacionales con el manejo de herramientas informáticas y cómo se considera esta competencia como clave y básica en el currículo oficial.

Parece, por tanto, que en política educativa se ha avanzado positivamente en este aspecto. En cambio, si se analizan las evidencias obtenidas en el presente estudio, que indican que el simple empleo de herramientas informáticas en el aula no aporta efectos positivos significativos sobre el desarrollo de competencias informacionales, se puede concluir que los avances logrados en el contexto institucional en este sentido no han contribuido a una mejora real. Estos resultados son congruentes con los obtenidos en otras investigaciones relacionadas (Ertmer y Ottenbreit-Leftwich, 2010; Suárez Rodríguez, Almerich, Gargallo y Aliaga, 2013).

En este sentido, muchos estudios llevados a cabo en diversos países indican que los alumnos emplean las TIC en el aula de manera escasa, y que este empleo es poco significativo sobre la mejora del rendimiento académico (OCDE, 2005, 2011; Pifarré et al., 2009; Sigalés, Mominó, 
Meneses y Badia, 2009). Los resultados alcanzados en esta investigación parecen confirmar estas sospechas: Tanto en hombres como en mujeres se detectan efectos insignificantes de la variable frecuencia de empleo de herramientas informáticas sobre las variables habilidades en el manejo de herramientas informáticas y competencias informacionales autopercibidas.

Sin embargo, el porcentaje de varianza explicada de las competencias informacionales en ambos grupos sostiene la existencia de una íntima relación entre estas y las TIC (Jarson, 2010; O'Farrill, 2008; Pifarré et al., 2009). Dado que el simple empleo de herramientas informáticas en el aula no facilita el desarrollo de competencias informacionales, se verifica la necesidad de una formación del profesorado en TIC, más que técnica, pedagógica (Almerich et al., 2013).

Entrando más concretamente en el tema que nos ocupa en este estudio, los datos obtenidos parecen confirmar en parte la hipótesis planteada. Una vez llevados a cabo los análisis estadísticos que descartan la existencia de factores indirectos que afectan a los resultados obtenidos en el análisis de vías, se muestra cómo existen diferencias significativas entre hombres y mujeres en lo que respecta a los efectos que tiene la frecuencia en el empleo de herramientas informáticas en casa sobre las competencias informacionales autopercibidas. Aunque parece que tanto hombres como mujeres emplean herramientas informáticas con la misma frecuencia en casa, parece que las diferentes tipologías de empleo que realizan de esas herramientas tienen diferentes efectos sobre el desarrollo de las competencias informacionales. Así, la tendencia de las diferencias encontradas indica que en los ambientes educativos no formales e informales, la manera en que emplean los hombres las herramientas informáticas facilita en mayor medida el desarrollo de competencias informacionales, que la manera en que lo hacen las mujeres. Sin embargo, este factor no parece contribuir en mejores indicadores de competencia informacional autopercibida en hombres que en mujeres (no se obtienen diferencias significativas en la prueba de diferencia de medias).

Por otro lado, como era de esperar a nivel teórico, la frecuencia en el empleo de herramientas informáticas en el aula y la propia destreza en este manejo, no causan efectos diferentes sobre la explicación de las competencias informacionales en función del sexo.

Otros estudios llevados a cabo al respecto parecen refrendar que «hombres y mujeres no se relacionan de la misma forma con el empleo de las TIC» (Iglesias Fernández et al., 2010: 105). Así, aunque la evidencia científica parece indicar que la relación con las TIC es diferente en hombres que en mujeres (Almerich et al., 2013; GargalloCastel et al., 2010; Iglesias Fernández et al., 2012; Ruiz Palomero y Sánchez Rodríguez, 2010) y que el desarrollo de competencias informacionales se comporta de manera diferenciada en ambos sexos, no se ha demostrado que el hecho de ser hombre o mujer facilite el desarrollo de competencias informáticas y/o informacionales.

Lo que el presente estudio sí parece indicar, es que existen ciertos factores atribuibles al género (diferentes perfiles de manejo de herramientas informáticas) que determinan el modo en el que se desarrollan las competencias informacionales. Así, cobra gran interés y relevancia el estudio específico de estos factores y su toma en consideración a la hora de afrontar procesos de enseñanza-aprendizaje que incluyan el desarrollo de competencias informacionales.

No debemos olvidar, no obstante, la limitación temporal del presente estudio, que muestra información recogida puntualmente en 2009. Se haría necesario, con el objeto de obtener una medida más fiable del estado actual de los estudiantes en este sentido, el estudio del estado de la cuestión más que transversal, longitudinal, de manera que aporte una idea de la evolución de los estudiantes en lo que al dominio de las competencias informacionales y en el manejo de herramientas informáticas se refiere. 
Así mismo, emergen en la presente investigación otras limitaciones, sobre todo en lo que se refiere a la evaluación de competencias mediante diseños ex post facto con instrumentos basados en la autopercepción. De cara a futuras investigaciones, por tanto, se hace necesaria la obtención de medidas objetivas, mediante instrumentos validados, del nivel de desarrollo de los estudiantes en competencias informacionales, y su análisis y valoración desde enfoques vinculados a diseños más experimentales.

Por último, y de cara a futuras investigaciones, se considera de interés el estudio de las diferencias concretas de género en esta relación con las TIC, la explicitación de las diferentes tipologías o perfiles de empleo de estas herramientas en función del género y, por último, la detección de los perfiles concretos que contribuyen en mayor medida en la explicación de la variabilidad del constructo competencias informacionales. De esta manera, se contribuiría en la explicación de algunos de los factores contribuyentes al desarrollo de las competencias informacionales y en una mayor aproximación a las diferencias reales de género, en lo que respecta al empleo de las TIC en los ambientes educativos no formales e informales.

\section{Referencias bibliográficas}

Backhoff Escudero, E. y GonzÁlez-Montesinos, M. J. (2012). Evidencias de validez del cuestionario para docentes del Estudio Internacional Sobre Enseñanza y Aprendizaje (TALIS 2009). Bordón, 64 (2), 173-194.

Bauman, Z. (2005). Education in Liquid Modernity. Review of Education, Pedagogy y Cultural Studies, 27 (4), 303-317.

Boletín Oficial del Estado (2007). Orden ECI/2220/2007, de 12 de julio, por la que se establece el currículo y se regula la ordenación de la Educación secundaria obligatoria. BOE $\mathrm{n}^{\mathrm{o}} 174$ (pp. 31680-31828).

Bollen, K. A. (1989). Structural Equations with Latent Variables. Wiley-Interscience.

BRowne, M. (1984). Asymptotically distribution-free methods for the analysis of covariance structures. The British journal of mathematical and statistical psychology, 37, 62-83.

Calvo Bernardino, A. y Mingorance Arnáiz, C. (2010). Evaluación continua de conocimientos vs. de competencias: Resultados de la aplicación de dos métodos valorativos diferentes. Revista de Investigación Educativa, 28 (2), 361-383.

Castells, M. (1999). La era de la información: economía, sociedad y cultura. La sociedad Red (vol. 1). Madrid: Alianza Editorial.

Catts, R. y LAU, J. (2009). Hacia unos Indicadores de Alfabetización Informacional. Madrid: Ministerio de Cultura.

Corder, G. W. y Foreman, D. I. (2009). Nonparametric Statistics for Non-Statisticians: A Step-by-Step Approach. New Jersey: Wiley

Crue-Tic y Rebiun. (2009). Competencias informáticas e informacionales en los estudios de grado. Recuperado a partir de http://crue-tic.uji.es/index.php?option=com_remositoryyItemid=28yfun $\mathrm{c}=$ startdownyid $=226$

De Miguel Díaz, M. (2010). La renovación del sistema educativo a partir de las competencias básicas. Organización y Gestión Educativa: Revista del Fórum Europeo de Administradores de la Educación, 18 (4), 394-402.

Diario Oficial de la Unión Europea (2006). Recomendación del Parlamento Europeo y del Consejo de 18 de diciembre de 2006 sobre las competencias clave para el aprendizaje permanente. Bruselas: Parlamento Europeo y Consejo de la Unión europea. Recuperado a partir 
de http://eur-lex.europa.eu/LexUriServ/site/es/oj/2006/1_394/1_39420061230es00100018. pdf

Ertmer, P. A. y Ottenbreit-Leftwich, A. T. (2010). Teacher Technology Change: How Knowledge, Confidence, Beliefs, and Culture Intersect. Journal of Research on Technology in Education, 42 (3), 255-284.

Fernández BAllesteros, R. (2004). Self-report questionnaires. En: S. N. Haynes, y E. M. Heiby (eds.), Comprehensive handbook of psychological assessment: Behavioral assessmen., (pp. 194-221). Hoboken, N. J., US: John Wiley \& Sons Inc.

Gargallo-Castel, A.; Esteban-Salvador, L. y Pérez Sanz, J. (2010). Impact of Gender in Adopting and Using ICTs in Spain. Journal of Technology Management y Innovation, 5 (3), 120-128.

HAWKInS, D. (1982). Topics in applied multivariate analysis. Cambridge [etc.]: Cambridge University Press. Hedges, L. (1982). Statistical methodology in meta-analysis. Princeton N. J.: ERIC.

Iglesias Fernández, C.; Llorente Heras, R. y Dueñas Fernández, D. (2010). Diferencias de género en el empleo TIC. Cuadernos de Economía, 33 (92), 105-137. doi: 10.1016/S02100266(10)70066-2

JARSON, J. (2010). Information literacy and higher education. College and Research Libraries News, $71(10), 534-538$.

JONES, B., y GofF, M. (2011). Learning to live with data deluge and what that means for educators. Teoría de la Educación: Educación y Cultura en la Sociedad de la Información, 12 (1), 9-27.

Jornet, J. M.; GonZÁlez-Such, J. y Perales, M. J. (2012). Diseño de cuestionarios de contexto para la evaluación de sistemas educativos: optimización de la medida de constructos complejos. Bordón, 64 (2), 89-110.

Kerlinger, F. y LeE, H. (2002). Investigación del comportamiento, métodos de investigación en Ciencias Sociales (4a ed.). México: McGrawHill.

KLINE, R. (2005). Principles and practice of structural equation modeling. New York: Guilford Press.

Mackey, T. P. y Jacobson, T. E. (2011). Reframing Information Literacy as a Metaliteracy. College y Research Libraries, 72 (1), 62-78.

Mateo Andrés, J. (2004). La investigación ex post-facto. En: R. Bisquerra Alzina (ed.), Metodología de la investigación educativa (pp. 196-230). Madrid: La Muralla.

Morales Vallejo, P.; Urosa, S. y Blanco, A. (2003). Construcción de escalas de actitudes tipo likert: una guía práctica. Madrid: La Muralla.

Mullola, S.; Jokela, M.; Ravaja, N.; Lipsanen, J.; Hintsanen, M.; Alatupa, S. y Keltikangas-Jarvinen, L. (2011). Associations of student temperament and educational competence with academic achievement: The role of teacher age and teacher and student gender. Teaching and Teacher Education, 27 (5), 942-951. doi:10.1016/j.tate.2011.03.005

MuthÉn, B. y Kaplan, D. (1985). A comparison of some methodologies for the factor analysis of non-normal Likert variables. British Journal of Mathematical and Statistical Psychology, 38 (2), 171-189.

O'FARrill, R. (2008). Information Literacy and Knowledge Management: Preparations for an Arranged Marriage. Libri, 58 (3), 155-171.

OCDE (2005). Are Students Ready for a Technology-Rich World? What PISA Studies Tell Us (Programme for International Student Assessment). Paris: OECD.

OCDE (2011). PISA 2009 Results: Students On Line. Digital technologies and performance. Paris: OECD. Recuperado a partir de http://dx.doi.org/10.1787/9789264112995-en

Ohland, M.; Brawner, C.; Camacho, M.; Layton, R.; Long, R.; Lord, S. y Wasburn, M. (2011). Race, Gender, and Measures of Success in Engineering Education. Journal of Engineering Education, 100 (2), 225-252. 
Autoevaluación de competencias informacionales en educacion secundaria: propuesta de modelo causal...

Pifarré, M.; Sanuy, J.; Vendrell, C. y Gòdia, S. (2009). Internet en la educación secundaria: pensar, buscar y construir conocimiento en la red. Lleida: Milenio.

Pinto, M. (2009). Design of the IL-HUMASS survey on information literacy in higher education: A self-assessment approach. Journal of Information Science, 36 (1), 86-103.

Rodríguez Conde, M. J.; Olmos Migueláñez, S. y Martínez Abad, F. (2012). Propiedades métricas y estructura dimensional de la adaptación española de una escala de evaluación de competencia informacional autopercibida (IL-HUMASS). Revista de Investigación Educativa, 30 (2), 347-365.

Rodríguez Conde, M. J.; Olmos Migueláñez, S.; Pinto Molina, M.; Martínez Abad, F. y García RiAzA, B. (2011). Informational Literacy And Information And Communication Technologies Use By Secondary Education Students In Spain: A Descriptive Study. Contemporary Issues in Education Research (CIER), 4 (4), 1-12.

Ruiz Palomero, J. y Sánchez Rodríguez, J. (2010). El género como factor influyente en la estrategia para integrar las TIC en la práctica docente. Pixel-Bit. Revista de Medios y Educación, 37, 67-76.

Schmidt, V.; Barreyro, J. P. y Maglio, A. L. (2010). Escala de evaluación del funcionamiento familiar FACES III: ¿Modelo de dos o tres factores? Escritos de Psicología, 3 (2), 30-36.

Sigalés, C.; Mominó, J. M.; Meneses, J. y BAdia, A. (2009). La integración de Internet en la educación escolar española: situación actual y perspectivas de futuro. Barcelona: Planeta.

SuÁrez Rodríguez, J. M.; Almerich, G.; Gargallo, B. y Aliaga, F. M. (2013). Las competencias del profesorado en TIC: estructura básica. Educación XX1, 16 (1), 39-62.

ValimaA, J. y Hoffman, D. (2008). Knowledge Society Discourse and Higher Education. Higher Education: The International Journal of Higher Education and Educational Planning, 56 (3), 265-285.

Weiner, S. A. (2010). Information Literacy: A neglected core competency. Educase Quarterly, 33 (1). Recuperado a partir de http://www.educause.edu/EDUCAUSE+Quarterly/EDUCAUSEQuarterlyMagazineVolum/InformationLiteracyANeglectedC/199382

Williamson, K. (2009). Information behavior of people in the fourth age: Implications for the conceptualization of information literacy. Library E Information Science Research, 31 (2), 76-83.

\section{Abstract}

\section{Information skills self-assessment in secondary education. A causal model proposal from a gender} perspective

INTRODUCTION. The inclusion of key skills related to information management in national and international basic education curricula responds to social change related to the impact of information and communication technologies. There are also a number of studies that find gender differences in the acquisition of computer skills. Given the close relationship that exists between information skills and technologies, this study analyzes gender differences in the development of these skills by designing a causal model. METHODS. The starting point is a representative sample of 1,162 secondary education students aged between 14 and 16 years from schools in the Spanish region of Castilla y León. RESULTS. The research findings obtained with this model indicate a good fit in both groups. The hypothesis testing of relationships proposed in the model shows that the effect exerted by the management tools profile at home (informal education environment) on information skills development varies according to gender. DISCUSSION. Given the importance that studying and defining 
these different profiles in the handling of computer tools has, discussion centers on the influence of these profiles on the learning of information skills.

Key words: Information and communication technologies, Information skills, Informal education, Structural equations modeling, Gender studies.

\section{Résumé}

Auto-évaluation des compétences informationnelles dans l'enseignement secondaire. Proposition d'un modèle causal à partir d'une perspective de genre

Linclusion de compétences clés liées à la gestion de l'information dans le curriculum de l'éducation de base au niveau national et international, répond à un changement social lié à l'impact des technologies de l'information et de la communication. Aussi, ils se multiplient les études de genre qui trouvent différences dans l'acquisition de compétences informatiques en fonction du sexe. En outre, il existe une relation étroite entre les compétences en recherche d'information et les technologies. Cette étude analyse les différences entre les sexes dans le développement de ces compétences sur le conception d'un modèle causal. Le point de départ est un échantillon représentatif de 1162 étudiants de l'enseignement secondaire âgés entre 14 et 16 ans des écoles de la région espagnole de Castilla y León. Les résultats obtenus indiquent bon ajustement dans les deux groupes. Le test d'hypothèse des relations proposées dans le modèle montre que l'effet exercé par le profil d'outils de gestion à la maison (milieu de l'éducation informelle), sur le développement des compétences en recherche d'information est différente selon le sexe. Enfin, la discussion est présentée sur l'influence des technologies de l'information et de la communication dans l'apprentissage de compétences en recherche d'information et de l'étude et la définition des profils différents dans le traitement de ces outils.

Mots clés: Technologies de l'information et de la communication, Compétence en recherche d'information, Éducation diffuse, Modélisation des équations structurelles, Études de genre.

\section{Perfil profesional de los autores}

\section{María José Rodríguez Conde}

Profesora Titular de Universidad y directora del instituto de investigación «Instituto Universitario de Ciencias de la Educación» (Universidad de Salamanca). Participante como investigadora e investigadora principal, en proyectos de investigación competitivos a nivel nacional e internacional. Posee numerosas publicaciones en revistas de impacto en el ámbito de la evaluación educativa.

Correo electrónico de contacto: mjrconde@usal.es

\section{Susana Olmos Migueláñez}

Ayudante Doctora y actualmente Secretaria Académica del Dpto. de Didáctica, Organización y Métodos de Investigación. Experta en e-evaluación y procesos de evaluación formativa, ha participado 
como investigadora en varios proyectos de investigación competitivos. Posee publicaciones en varias revistas nacionales e internacionales de impacto.

Correo electrónico de contacto: solmos@usal.es

\section{Fernando Martínez Abad}

Ayudante en el Área de Métodos de Investigación y Diagnóstico en Educación de la Universidad de Salamanca. Doctor en Ciencias de la Educación, ha participado como investigador en proyectos de investigación nacionales I+D. Coautor en varias publicaciones nacionales e internacionales relacionadas con la evaluación y desarrollo de competencias básicas en la educación obligatoria.

Correo electrónico de contacto: fma@usal.es 